\title{
Note on Neighborhoods of Some Classes of Analytic Functions with Negative Coefficients
}

\author{
Irina Dorca, ${ }^{1}$ Mugur Acu, $^{2}$ and Daniel Breaz ${ }^{3}$ \\ ${ }^{1}$ Department of Mathematics, University of Piteşti, 110040 Argeş, Romania \\ 2 Department of Mathematics, "Lucian Blaga" University of Sibiu, 550024 Sibiu, Romania \\ ${ }^{3}$ Department of Mathematics, "1 Decembrie 1918" University of Alba Iulia, 510009 Alba Iulia, Romania \\ Correspondence should be addressed to Mugur Acu, acu_mugur@yahoo.com
}

Received 12 April 2011; Accepted 15 May 2011

Academic Editor: R. Avery

Copyright (C) 2011 Irina Dorca et al. This is an open access article distributed under the Creative Commons Attribution License, which permits unrestricted use, distribution, and reproduction in any medium, provided the original work is properly cited.

In this paper, we prove several inclusion relations associated with the $(n, \delta)$ neighborhoods of some subclasses of starlike and convex functions with negative coefficients.

\section{Introduction}

Let $\mathscr{L}(U)$ be the set of functions which are regular in the unit disc $U$,

$$
A=\left\{f \in \mathscr{H}(U): f(0)=f^{\prime}(0)-1=0\right\}
$$

and $S=\{f \in A: f$ is univalent in $U\}$.

In [1], the subfamily $T$ of $S$ consisting of functions $f$ of the form

$$
f(z)=z-\sum_{j=2}^{\infty} a_{j} z^{j}, \quad a_{j} \geq 0, j=2,3, \ldots, z \in U,
$$

was introduced, where

$$
T=\left\{f \in S: f(z)=z-\sum_{j=2}^{\infty} a_{j} z^{j}, a_{j} \geq 0, j \geq 2, z \in U\right\} .
$$


For $f(z)$ belonging to $A$, Sălăgean [2] has introduced the following operator called the Sălăgean operator:

$$
D^{0} f(z)=f(z), \quad D^{1} f(z)=D f(z)=z f^{\prime}(z), \quad D^{n} f(z)=D\left(D^{n-1} f(z)\right), \quad n \in \mathbb{N}^{*}
$$

The object of the present paper is to derive some properties of neighborhoods for some subclasses of analytic functions with negative coefficients, which we have already studied.

\section{Preliminary Results}

Remark 2.1. In [3], we have introduced the following operator concerning the functions of form (1.2):

$$
D_{\lambda}^{\beta}: A \longrightarrow A, \quad D_{\lambda}^{\beta} f(z)=z-\sum_{j=n+1}^{\infty}[1+(j-1) \lambda]^{\beta} a_{j} z^{j}
$$

Definition 2.2 (see [3]). Let $f \in T, f(z)=z-\sum_{j=2}^{\infty} a_{j} z^{j}$, and $a_{j} \geq 0, j=2,3, \ldots, z \in U$. We say that $f$ is in the class $T L_{\beta}(\alpha)$ if

$$
\operatorname{Re} \frac{D_{\lambda}^{\beta+1} f(z)}{D_{\lambda}^{\beta} f(z)}>\alpha, \quad \alpha \in[0,1), \lambda \geq 0, \beta \geq 0, z \in U
$$

Theorem 2.3 (see [3]). Let $\alpha \in[0,1), \lambda \geq 0, n \in \mathbb{N}^{*}$, and $\beta \geq 0$. The function $f \in A(n)$ of the form (1.2) is in the class $T^{*} L_{\beta, n}(\alpha)$ if and only if

$$
\sum_{j=n+1}^{\infty}\left[(1+(j-1) \lambda)^{\beta}(1+(j-1) \lambda-\alpha)\right] \cdot a_{j}<1-\alpha
$$

Remark 2.4. Using the condition (1.3), we can to prove that

$$
T^{*} L_{\beta+1, n}(\alpha) \subset T^{*} L_{\beta, n}(\alpha),
$$

$\beta \geq 0, \alpha \in[0,1), \lambda \geq 0$, and $n \in \mathbb{N}^{*}$.

Definition 2.5 (see [4]). Let $f \in T, f(z)=z-\sum_{j=2}^{\infty} a_{j} z^{j}$, and $a_{j} \geq 0, j=2,3, \ldots, z \in U$. We say that $f$ is in the class $T^{c} L_{\beta}(\alpha)$ if

$$
\operatorname{Re} \frac{D_{\lambda}^{\beta+2} f(z)}{D_{\lambda}^{\beta+1} f(z)}>\alpha, \quad \alpha \in[0,1), \lambda \geq 0, \beta \geq 0, z \in U
$$


Theorem 2.6 (see [4]). Let $\alpha \in[0,1), \lambda \geq 0$, and $\beta \geq 0$. The function $f \in T$ of the form (1.2) is in the class $T^{c} L_{\beta}(\alpha)$ if and only if

$$
\sum_{j=2}^{\infty}\left[(1+(j-1) \lambda)^{\beta+1}(1+(j-1) \lambda-\alpha)\right] a_{j}<1-\alpha .
$$

Remark 2.7. Using the condition (2.2), we can prove that

$$
T^{c} L_{\beta+1, n}(\alpha) \subset T^{c} L_{\beta, n}(\alpha),
$$

$\beta \geq 0, \alpha \in[0,1), \lambda \geq 0$, and $n \in \mathbb{N}^{*}$.

Let $A(n)$ be the class of functions $f(z)$ of the form

$$
f(z)=z-\sum_{k=n+1}^{\infty} a_{k} z^{k}, \quad\left(a_{k} \geq 0 ; n \in \mathbb{N}-\{0\}\right)
$$

which are analytic in the open unit disk $U=\{z:|z|<1\}$. For any $f(z) \in A(n)$ and $\delta \geq 0$, we define

$$
N_{n, \delta}=\left\{g \in A(n): g(z)=z-\sum_{k=n+1}^{\infty} b_{k} z^{k}, \sum_{k=n+1}^{\infty} k \cdot\left|a_{k}-b_{k}\right| \leq \delta\right\},
$$

which was called $(n, \delta)$-neighborhood of $f(z)$. So, for $e(z)=z$, we observe that

$$
N_{n, \delta}=\left\{g \in A(n): g(z)=z-\sum_{k=n+1}^{\infty} b_{k} z^{k}, \sum_{k=n+1}^{\infty} k \cdot\left|b_{k}\right| \leq \delta\right\} .
$$

The concept of neighborhoods was first introduced by Goodman in [5] and then generalized by Ruscheweyh in [6].

We propose to investigate the $(n, \delta)$-neighborhoods of the subclasses $T^{*} L_{\beta, n}(\alpha)$ and $T^{c} L_{\beta, n}(\alpha)$ of the class $A(n)$ of normalized analytic functions in $U$ with negative coefficients, where $T^{*} L_{\beta, n}(\alpha)$ is the subclass of $n$-starlike functions with negative coefficients of order $\alpha$ and type $\beta$ introduced in [3] and $T^{c} L_{\beta, n}(\alpha)$ is the subclass of $n$-convex functions with negative coefficients of order $\alpha$ and type $\beta$ studied in [4].

\section{Main Results}

We start by considering the linear operator (2.1) and conclude the study with several general inclusion relations associated with the $(n, \delta)$ neighborhoods for some subclasses of starlike and convex functions with negative coefficients.

Using the inequality (1.3) from Definition 2.5 and the inequality (2.1) from Definition 2.2, we obtain the subclasses $T^{*} L_{\beta, n}(\alpha)$, and $T^{c} L_{\beta, n}(\alpha)$ and from Theorem 2.3, we derive the corresponding results. 
Theorem 3.1. Let

$$
\delta=\frac{(1-\alpha)(n+1)}{(1+n \lambda)^{\beta}(1+n \lambda-\alpha)}
$$

where $\alpha \in[0,1), \lambda \geq 0, \beta \geq 0$, and $n \in \mathbb{N}^{*}$; then

$$
T^{*} L_{\beta, n}(\alpha) \subset N_{n, \delta}(e)
$$

Proof. For $f(z) \in T^{*} L_{\beta, n}(\alpha)$ and making use of the condition (2.2), we obtain $\left[(1+n \lambda)^{\beta}(1+\right.$ $n \curlywedge-\alpha)] \sum_{j=n+1}^{\infty} a_{j}<1-\alpha$, so that

$$
\sum_{j=n+1}^{\infty} a_{j}<\frac{1-\alpha}{(1+n \lambda)^{\beta}(1+n \lambda-\alpha)} .
$$

On the other hand, we also find from (2.2) and (3.3) that

$$
\begin{gathered}
\sum_{j=n+1}^{\infty}\left[(1+(j-1) \lambda)^{\beta}(1+(j-1) \mathcal{L}-\alpha)\right] a_{j}<1-\alpha, \\
(1+n \lambda)^{\beta} \sum_{j=n+1}^{\infty}(1+(j-1) \lambda-\alpha) a_{j}<1-\alpha, \\
\lambda(1+n \lambda)^{\beta} \sum_{j=n+1}^{\infty} j \cdot a_{j}<1-\alpha-\left[(1-\lambda-\alpha)(1+n \lambda)^{\beta}\right] \cdot \sum_{j=n+1}^{\infty} a_{j} .
\end{gathered}
$$

Thus,

$$
\sum_{j=n+1}^{\infty} j \cdot a_{j}<\frac{(1-\alpha)(n+1)}{(1+n \lambda)^{\beta}(1+n \mathcal{\lambda}-\alpha)}=\delta,
$$

which in view of definition (2.10), proves Theorem 3.1.

Remark 3.2. If $\lambda=1$ and $\beta=1$, we obtain that $\sum_{j=n+1}^{\infty} j \cdot a_{j}<(1-\alpha) /(1+n-\alpha)=\delta$ for $D_{1}^{1} f(z)=z f^{\prime}(z), f(z) \in T_{n}^{*}(\alpha)$.

In a similar way, applying (2.5) instead of (2.2), we can prove the following.

Theorem 3.3. Let

$$
\delta=\frac{(1-\alpha)(n+1)}{(1+n \lambda)^{\beta+1}(1+n \lambda-\alpha)}
$$


where $\alpha \in[0,1), \lambda \geq 0, \beta \geq 0$, and $n \in \mathbb{N}^{*}$; then

$$
T^{c} L_{\beta, n}(\alpha) \subset N_{n, \delta}(e) .
$$

Consequently, we determine the neighborhood for each of the classes $T^{*(v)} L_{\beta, n}(\alpha)$ and $T^{c(v)} L_{\beta, n}(\alpha)$, which we define as follows. A function $f(z) \in A(n)$ defined by (2.8) is said to be in the class $T^{*(v)} L_{\beta, n}(\alpha)$ if there exists a function $g(z) \in T^{*} L_{\beta, n}(\alpha)$ such that

$$
\left|\frac{f(z)}{g(z)}-1\right|<1-\alpha, \quad z \in U, \alpha \in[0,1)
$$

Analogously, a function $f(z) \in A(n)$ defined by (2.8) is said to be in the class $T^{c(v)} L_{\beta, n}(\alpha)$ if there exists a function $g(z) \in T^{c} L_{\beta, n}(\alpha)$ such the inequality (3.8) holds.

Remark 3.4. If $\lambda=1$ and $\beta=1$, we obtain that $\sum_{j=n+1}^{\infty} j \cdot a_{j}<(1-\alpha) /((1+n)(1+n-\alpha))=\delta$ for $D_{1}^{1} f(z)=z f^{\prime}(z), f(z) \in T_{n}^{c}(\alpha)$.

Further, we consider the inclusion relations just studied and generalize them by taking into account the relation (2.9).

Theorem 3.5. If $g(z) \in T^{*} L_{\beta, n}(\alpha)$ and

$$
v=1-\frac{\delta \cdot\left[(1+n \lambda)^{\beta}(1+n \lambda-\alpha)\right]}{(n+1) \cdot\left[(1+n \lambda)^{\beta}(1+n \lambda-\alpha)-(1-\alpha)\right]},
$$

then

$$
N_{n, \delta}(g) \subset T^{*(v)} L_{\beta, n}(\alpha),
$$

$\alpha \in[0,1), \lambda \geq 0, \beta \geq 0$, and $n \in \mathbb{N}^{*}$.

Proof. Let $f(z) \in N_{n, \delta}(g)$. Making use of (2.9), we find that

$$
\sum_{j \geq n+1} j \cdot\left|a_{j}-b_{j}\right|<\delta, \quad n \in \mathbb{N}^{*}
$$

which readily implies the coefficients of inequality

$$
\sum_{j \geq n+1}\left|a_{j}-b_{j}\right|<\frac{\delta}{n+1}, \quad n \in \mathbb{N}
$$

Furthermore, since $g(z) \in T^{*} L_{\beta, n}(\alpha)$, we have

$$
\sum_{j \geq n+1} b_{j}<\frac{1-\alpha}{(1+n \lambda)^{\beta}(1+n \lambda-\alpha)}
$$


so that

$$
\left|\frac{f(z)}{g(z)}-1\right|<\frac{\sum_{j \geq n+1}\left|a_{j}-b_{j}\right|}{1-\sum_{j \geq n+1} b_{j}}<\frac{\delta \cdot\left[(1+n \lambda)^{\beta}(1+n \lambda-\alpha)\right]}{(n+1) \cdot\left[(1+n \lambda)^{\beta}(1+n \lambda-\alpha)-(1-\alpha)\right]}=1-v,
$$

provided that $v$ is given precisely by (3.9), which evidently completes our proof of Theorem 3.5.

Example 3.6. For a given $g(z)=z-\sum_{j \geq n+1} b_{j} z^{j} \in T^{*} L_{\beta, n}(\alpha), n \in \mathbb{N}, n>1$, we consider $f(z)=$ $z-\sum_{j \geq n+1} a_{j} z^{j} \in T$ with $a_{j}=(1-v) /\left(j^{2}(j-1)\right)+b_{j}, j \geq n+1, n \in \mathbb{N} \backslash\{0\},($ for $\beta=0, \lambda=1)$, where $1-v$ is given by (3.9).

Then we have that

$$
\sum_{j \geq n+1} j\left|a_{j}-b_{j}\right|=\sum_{j \geq n+1} j\left|\frac{1-v}{j^{2}(j-1)}\right|=(1-v)\left\{\sum_{j \geq n+1}\left(\frac{1}{j-1}-\frac{1}{j}\right)\right\}=1-v,
$$

where $1-v=\delta(1+n-\alpha) /(n(n+1))$.

Therefore, $f(z) \in N_{n, \delta}(g)$.

In a similar way, we can prove Theorem 3.7.

Theorem 3.7. If $g(z) \in T^{c} L_{\beta, n}(\alpha)$ and

$$
v=1-\frac{\delta \cdot\left[(1+n \lambda)^{\beta+1}(1+n \lambda-\alpha)\right]}{(n+1) \cdot\left[(1+n \lambda)^{\beta+1}(1+n \lambda-\alpha)-(1-\alpha)\right]},
$$

then

$$
N_{n, \delta}(g) \subset T^{c(v)} L_{\beta, n}(\alpha),
$$

$\alpha \in[0,1), \lambda \geq 0, \beta \geq 0$, and $n \in \mathbb{N}^{*}$.

Example 3.8. For a given $g(z)=z-\sum_{j \geq n+1} b_{j} z^{j} \in T^{c} L_{\beta, n}(\alpha), n \in \mathbb{N}, n>1$, we consider $f(z)=z-\sum_{j \geq n+1} a_{j} z^{j} \in T$ with $a_{j}=(1-v) /\left(j^{2}(j-1)\right)+b_{j}, j \geq n+1, n \in \mathbb{N} \backslash\{0\},($ for $\beta=0$, $\mathcal{l}=1$ ), where $1-v$ is given by (3.16).

Then we have that

$$
\sum_{j \geq n+1} j\left|a_{j}-b_{j}\right|=\sum_{j \geq n+1} j\left|\frac{1-v}{j^{2}(j-1)}\right|=(1-v)\left\{\sum_{j \geq n+1}\left(\frac{1}{j-1}-\frac{1}{j}\right)\right\}=1-v,
$$

where $1-v=\delta(1+n-\alpha) /(n(n+1))$.

Therefore, $f(z) \in N_{n, \delta}(g)$. 


\section{Acknowledgment}

This work was partially supported by the strategic project POSDRU 107/1.5/S/77265, inside POSDRU Romania 2007-2013 cofinanced by the European Social Fund-Investing in People.

\section{References}

[1] H. Silverman, "Univalent functions with negative coefficients," Proceedings of the American Mathematical Society, vol. 51, pp. 109-116, 1975.

[2] G. S. Sălăgean, "Subclasses of univalent functions," in Complex Analysis_Fifth Romanian-Finnish Seminar, Part 1 (Bucharest, 1981), vol. 1013 of Lecture Notes in Mathematics, pp. 362-372, Springer, Berlin, Germany, 1983.

[3] M. Acu, I. Dorca, and S. Owa, "On some starlike functions with negative coefficients," Acta Universitatis Apulensis, Special Issue, Alba Iulia. In press.

[4] M. Acu, I. Dorca, and D. Breaz, "About some convex functions with negative coefficients," Acta Universitatis Apulensis, no. 14, 2007.

[5] A. W. Goodman, "Univalent functions and nonanalytic curves," Proceedings of the American Mathematical Society, vol. 8, pp. 598-601, 1957.

[6] S. Ruscheweyh, "Neighborhoods of univalent functions," Proceedings of the American Mathematical Society, vol. 81, no. 4, pp. 521-527, 1981. 


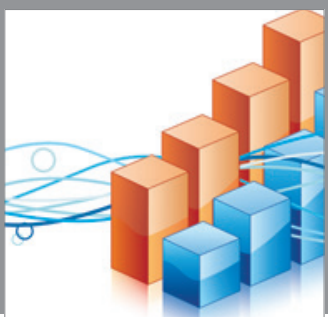

Advances in

Operations Research

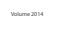

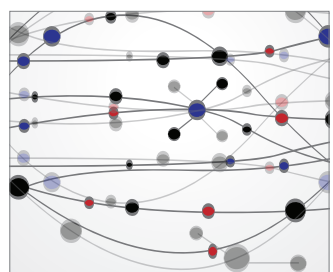

\section{The Scientific} World Journal
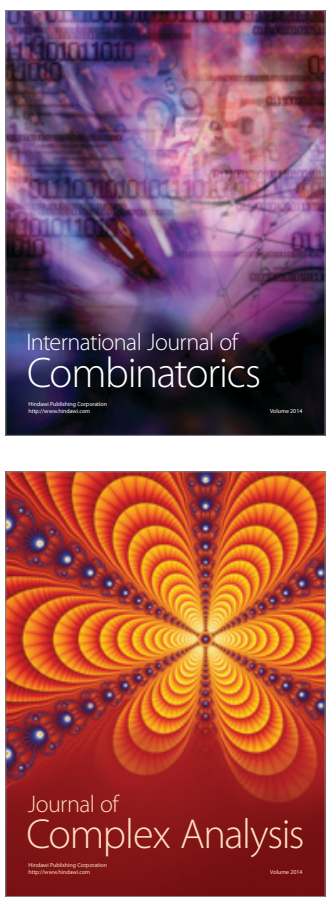

International Journal of

Mathematics and

Mathematical

Sciences
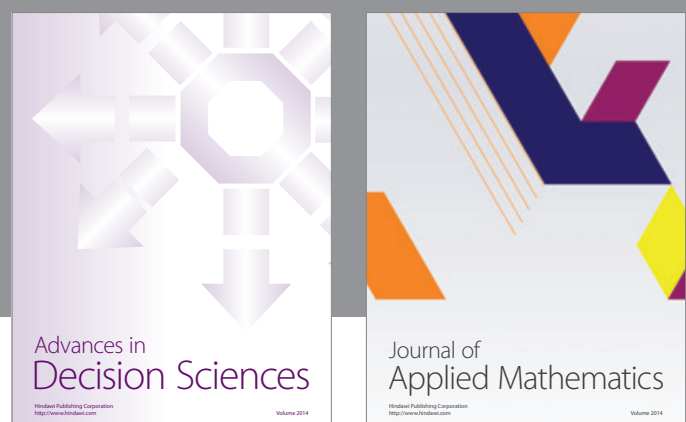

Journal of

Applied Mathematics
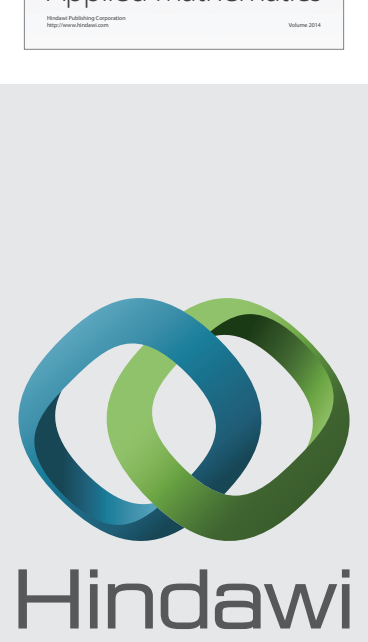

Submit your manuscripts at http://www.hindawi.com
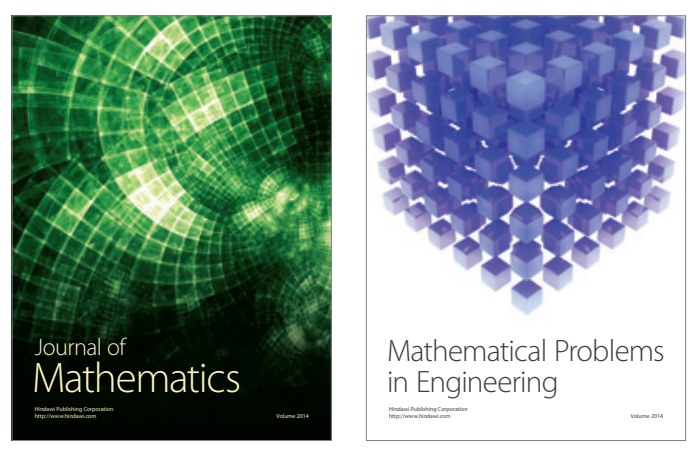

Mathematical Problems in Engineering
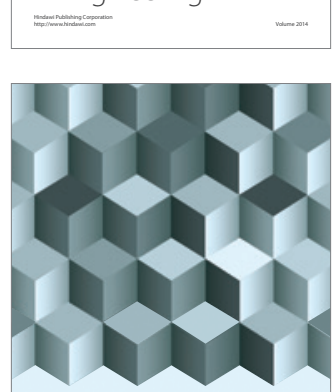

Journal of

Function Spaces
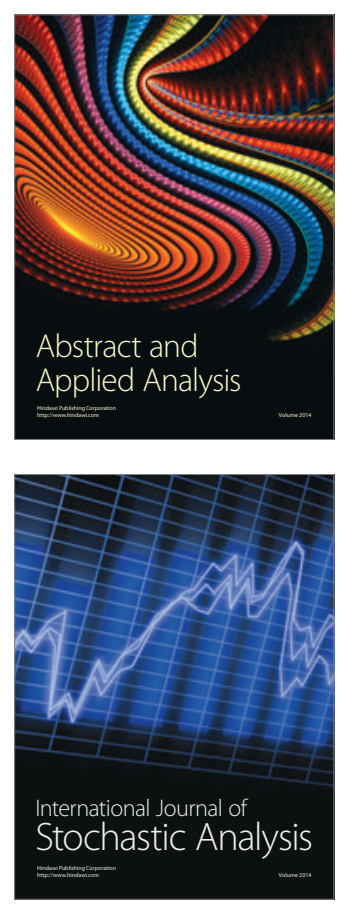

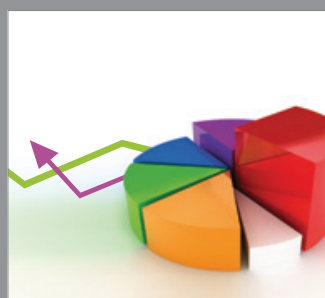

ournal of

Probability and Statistics

Promensencen
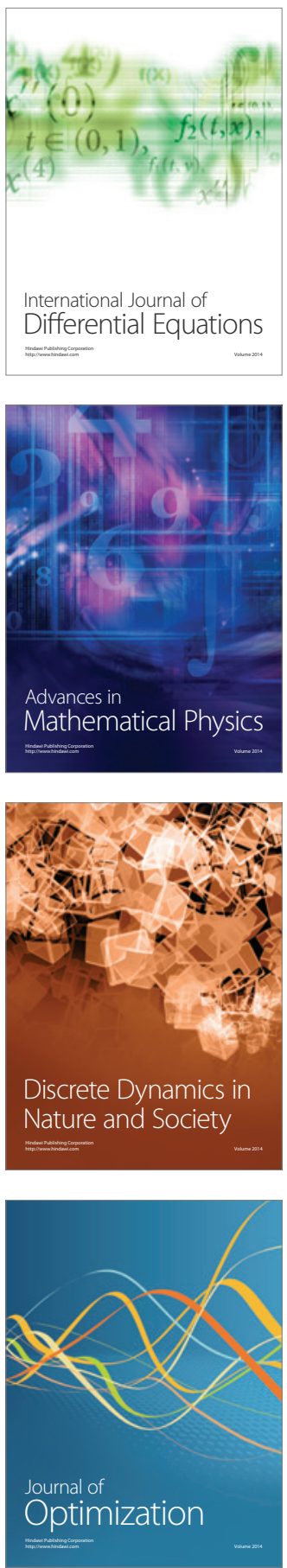